\title{
STRONG (WEAK) VV-DOMINATING SET OF A GRAPH
}

\author{
Sayinath Udupa ${ }^{1 a}$, R. S. Bhat ${ }^{* 2 a}$ \\ a,Department of Mathematics, Manipal Institute of Technology, A Constituent unit of Manipal Academy Of Higher \\ Education, Manipal, 576104, India. Email: sayinath.udupa@manipal.edu ${ }^{1 \mathrm{a}}$; rs.bhat@manipal.edu ${ }^{2 a}$ \\ *Corresponding author: rs.bhat@ manipal.edu \\ Received: $2^{\text {nd }}$ Feb 2018 \\ Accepted: $24^{\text {th }}$ Jul 2019 \\ Published: $31^{\text {st }}$ Dec 2019
}

DOI: https://doi.org/10.22452/mjs.vol38no3.3

ABSTRACT Two vertices $u, w \in V$ vv-dominate each other if they incident on the same block. A vertex $u \in V$ strongly vv-dominates a vertex $w \in V$ if $u$ and $w$, vv-dominate each other and $d_{v v}(u) \geq d_{v v}(w)$. A set of vertices is said to be strong vv-dominating set if each vertex outside the set is strongly vv-dominated by at least one vertex inside the set. The strong vv-domination number $\gamma_{s v v}(G)$ is the order of the minimum strong vv-dominating set of $G$. Similarly weak vvdomination number $\gamma_{w v v}(G)$ is defined. We investigate some relationship between these parameters and obtain Gallai's theorem type results. Several upper and lower bounds are established. In addition, we characterize the graphs attaining some of these bounds.

Keywords: Strong (weak) vv-dominating sets, Strong (weak) vv-full sets.

Mathematics Subject Classification: 05C69

\section{INTRODUCTION}

For notations and terminologies refer (Harary, 1969; West, 1996). Let $G$ be a graph of order $p$ and size $q$. The set of vertices is said to be an independent set if no two vertices in the set are adjacent. Then the maximum order of the independent set is called independence number $\beta_{0}(G)$. If a vertex $v$ is incident with an edge $x$ then we say that $v$ and $x$ cover each other. The minimum number of vertices required to cover all the edges of $G$ is called vertex covering number $\alpha_{0}(G)$. These two parameters are related by $\alpha_{0}(G)+\beta_{0}(G)=$ $p$ which is now referred as classical Gallai's Theorem (Gallai, 1959). Two vertices are said to dominate each other if they are adjacent. A set of vertices is a dominating set if each vertex outside the set is dominated by at least one vertex inside the set. Then the order of the minimum dominating set is called as domination number $\gamma(G)$. Similarly edge domination number $\gamma^{\prime}(G)$ is defined. The domination number is a well studied parameter in literature and for a survey refer (Berge, 1962; Haynes et al., 1998). Sampathkumar and Pushpa Latha (Sampathkumar et al., 1996) introduced strong (weak) dominating sets. Let $G$ be a graph and $u_{1} u_{2} \in E$. Then $u_{1}$ strongly dominates $u_{2}$ if $\operatorname{deg}\left(u_{1}\right) \geq \operatorname{deg}\left(u_{2}\right)$. A set of vertices is said to be strong dominating set (sd-set) if each vertex outside the set is strongly dominated by at least one vertex inside the set. The order of a minimum sd-set of $G$ is called strong domination number $\gamma_{s}(G)$. The strong domination is later studied 
in (Rautenbach , 1998 ; Hattingh et al., 1998; Henning , 1998). Similar to strong (weak) domination S. S. Kamath and R. S. Bhat (Kamath, 2007) studied strong (weak) independent sets. A vertex $u \in V$ is a cutvertex if $G-u$ is disconnected. A graph which has no cutvertex is called nonseparable. A maximal non-separable subgraph is a block of $G$. Let $B(G)$ and $C(G)$ respectively denote the set of all blocks and cutvertices of $G$. Let $m$ and $n$ denote the number of blocks and cutvertices of $G$. If a block $b \in B(G)$ contains a cutvertex $c \in$ $C(G)$ then we say that $b$ and $c$ incident to each other. Any two blocks in the graph are said to be adjacent if they share a vertex in common. On the other hand, any two cutvertices are adjacent if they incident on the same block. P. G. Bhat and R. S. Bhat (Bhat et al., 2013) defined vv-dominating sets. Two vertices $u_{1}, u_{2} \in V$ are vv-adjacent if they incident on the same block. Then vv-degree of a vertex $w, d_{v v}(w)$ is the number of vertices vvadjacent to $w$. Two vertices $u_{1}$ and $u_{2}$ are said to vv-dominate each other if they are vvadjacent. A set of vertices is called as the vvdominating set (VVD-set) if each vertex outside the set is vv-dominated by at least one vertex inside set. Then the order of minimum VVD-set is called vv-domination number $\gamma_{v v}(G)$. A set of vertices is said to be vv-full if each vertex in the set $\mathrm{vv}$-dominates at least one vertex outside the set. Then the order of the maximum vv-full set of the graph $G$ is called vv-full number $f_{v v}(G)$.

\section{STRONG VV-DEGREE AND WEAK VV-DEGREE OF A VERTEX}

A vertex $u \in V$ strongly (weakly) vvdominates a vertex $w \in V$ if $u$ and $w$ vvdominate each other and $d_{v v}(u) \geq$ $d_{v v}(w)\left(d_{v v}(u) \leq d_{v v}(w)\right)$. Strong (weak) vv-degree of a vertex $u$, denoted as $d_{s v v}(u)$ (denoted as $d_{w v v}(u)$ ) is the number of vertices strongly (weakly) vv-dominated by the vertex $u$. A vertex $u \in V$ regularly vvdominates a vertex $w \in V$ if $u$ and $w$, vvdominate each other and $d_{v v}(u)=d_{v v}(w)$. Regular vv-degree of the vertex $u$, denoted as $d_{r v v}(u)$ is the number of vertices regularly vv-dominated by the vertex $u$.

Example 2.1: In the Figure 1, the first, second and third elements of a vertex label represent strong vv-degree, weak vv-degree and regular vv-degree of the corresponding vertices respectively.

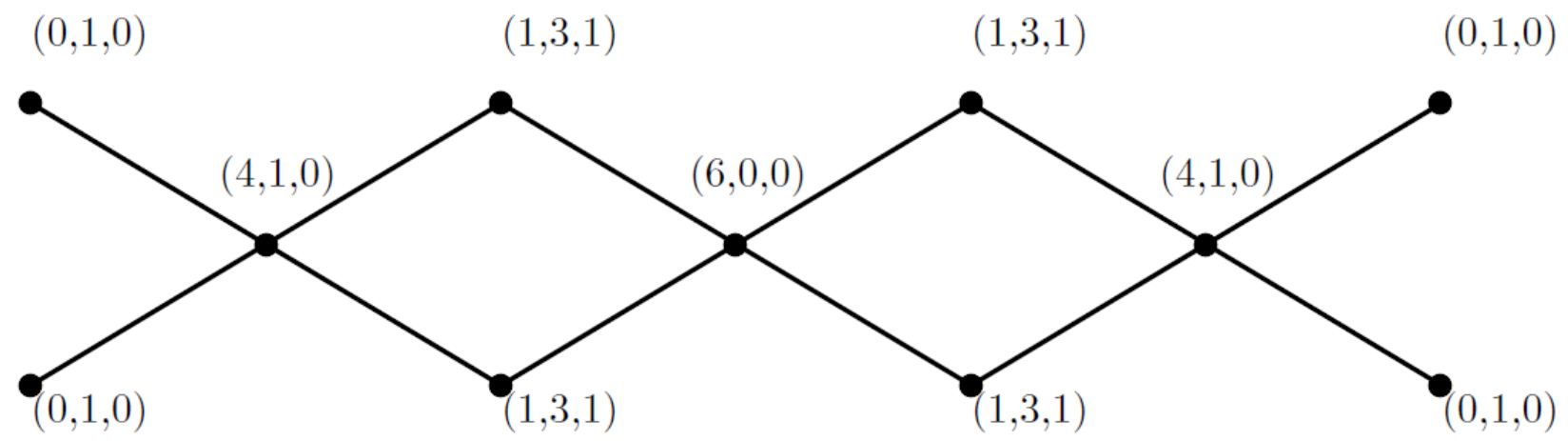

Figure 1. Strong vv-degree, weak vv-degree and regular vv-degree of a vertex. 
Proposition 2.1: Let $G$ be any graph and $u \in V$, then

$$
d_{v v}(u)=d_{s v v}(u)+d_{w v v}(u)-d_{r v v}(u)
$$

Proof: For any vertex $u \in V$, Let $A=$ number of vertices vv-dominated by $u, S=$ number of vertices strongly vv-dominated by $u, W=$ number of vertices weakly vv-dominated by $u$ and $R=$ number of vertices regularly vvdominated by $u$. We observe that $A=S \cup W$, $R=S \cap W$. Therefore $\quad d_{v v}(u)=|A|=$ $|W \cup S|=|W|+|S|-|W \cap S|=$ $d_{w v v}(u)+d_{s v v}(u)-d_{r v v}(u)$.

\section{VV-STRONG NUMBER, VV- WEAK NUMBER, VV- BALANCED AND VV- REGULAR NUMBER}

A vertex $u \in V$ is vv-strong (vvweak) if $\quad d_{v v}(u) \geq d_{v v}(w) \quad\left(d_{v v}(u) \leq\right.$ $\left.d_{v v}(w)\right)$ for every $w$ which is vv-adjacent to $u$. A vertex $u \in V$ is vv-balanced if it is neither vv-strong nor vv-weak. A vertex $u \in$ $V$ is vv-regular if it is both vv-strong and vvweak. A set $D \subseteq V$ is said to be vv-strong set, vv-weak set, vv-balanced set and vv-regular set of $G$ if each vertex in $D$ is vv-strong, vvweak, vv-balanced and vv-regular vertex of $G$ respectively. The vv-strong number $s_{v v}=$ $s_{v v}(G)$, the vv-weak number $w_{v v}=$ $w_{v v}(G)$, the vv-balanced number $b_{v v}=$ $b_{v v}(G)$, and vv-regular number $r_{v v}=$ $r_{v v}(G)$ is the order of a vv-strong, vv-weak, vv-balanced and vv-regular set of $G$ respectively. A vertex $u \in V$ is strictly vvstrong (strictly vv-weak) if $d_{v v}(u)>$ $d_{v v}(w)\left(d_{v v}(u)<d_{v v}(w)\right)$ for every $w$ vvadjacent to $u$.

Proposition 3.1: Let $G$ be any graph with p vertices, then

$$
s_{v v}(G)+w_{v v}(G)+b_{v v}(G)-r_{v v}(G)=p
$$

Proof: Let $G$ be the graph with $p$ vertices. Let $S_{v v}$ be the vv-strong set, $W_{v v}$ be the vv-weak set, $B_{v v}$ be the vv-balanced set and $R_{v v}$ be the vv-regular set. We have $S_{v v} \cap W_{v v}=R_{v v}$ by definition. Since any vv-balanced vertex is neither vV-strong nor vV-weak, therefore it cannot be either in $S_{v v}$ or in $W_{v v}$. Therefore $\left(S_{v v} \cup W_{v v}\right) \cap B_{v v}=\phi$. Also we have $V=$ $\left(S_{v v} \cup W_{v v}\right) \cup B_{v v}$. Then $|V|=p=\mid S_{v v} \cup$ $W_{v v}|+| B_{v v}|=| S_{v v}|+| W_{v v}|-| S_{v v} \cap$ $W_{v v}|+| B_{v v} \mid=s_{v v}(G)+w_{v v}(G)-$ $r_{v v}(G)+b_{v v}(G)$.

Proposition 3.2: For any graph $G=(V, E)$ with vertex $u \in V$.

i) $\quad u \in V$ is vv-strong if and only if $d_{v v}(u)=d_{s v v}(u)$

ii) $\quad u \in V$ is $v v$-weak if and only if $d_{v v}(u)=d_{w v v}(u)$

iii) $\quad u \in V$ is balanced if and only if $d_{v v}(u)<d_{v v}(v)$ and $d_{v v}(u)>d_{v v}(w)$ for some $v$ and $w, v v$-adjacent to $u$.

iv) $\quad u \in V$ is vv-regular if and only if $d_{v v}(u)=d_{s v v}(u)=d_{w v v}(u)=d_{r v v}(u)$.

v) $\quad u \in V$ is strictly $v v$-strong if and only if $d_{w v v}(u)=0$.

vi) $\quad u \in V$ is strictly $v v$-weak if and only if $d_{s v v}(u)=0$. 
Proof :

i) Let $u \in V$ be a vv-strong vertex. Then $d_{v v}(u) \geq d_{v v}(w)$ for every vertex $w$ vvadjacent to $u$. Therefore $N_{w v v}(u)=\{w \in V(G) \mid w$ is weakly vv dominated by the vertex $u\}=N_{r v v}(u)$.

Hence $d_{w v v}(u)=d_{r v v}(u)$. Then by Proposition 2.1, we have $d_{v v}(u)=d_{s v v}(u)$. Converse follows from the definition.

ii) can be proved with the similar argument and hence we omit the proof.

iii) Let $u \in V$ be a vv-balanced vertex. Suppose both the conditions does not hold, then $d_{v v}(u)=d_{v v}(w)$ for every vertex $w$ vvadjacent to $u$. Hence $u$ is a vv-regular vertex, a contradiction. Conversely, suppose both the conditions hold. Since $d_{v v}(u)<d_{v v}(v)$ for some $v$ which is vv-adjacent to $u$. From this we conclude that $u$ is not a vv-strong vertex. Similarly $u$ cannot be a vv-weak vertex as $d_{v v}(u)>d_{v v}(w)$ for some $w, v v$-adjacent to $u$. Then by definition, $u$ must be a vvbalanced vertex.

(iv), (v) and (vi) directly follow from the definition.

\section{STRONG (WEAK) VV- DOMINATING SETS OF A GRAPH}

A vertex $u \in V$ strongly (weakly) vvdominates a vertex $w \in V$ if $\mathrm{u}$ and $w$, vvdominates each other and $d_{v v}(u) \geq$ $d_{v v}(w)\left(d_{v v}(u) \leq d_{v v}(w)\right)$. A set of vertices is said to be strong vV-dominating set (SVVD-set) (weak vv-dominating set (WVVD-set)) if each vertex outside the set is strongly (weakly) vv-dominated by a vertex inside the set. The strong vv-domination number $\quad \gamma_{s v v}=\gamma_{s v v}(G) \quad$ (weak vvdomination number $\left.\gamma_{w v v}=\gamma_{w v v}(G)\right)$ is the order of the minimum SVVD-set (WVVDset) of $G$. A set of vertices is said to be strong vv-full set(SVVF-set) (weak vv-full set (WVVF-set)) if each vertex inside the set strongly (weakly) vv-dominates at least one vertex outside the set. The strong vv-full number $f_{s v v}=f_{s v v}(G)$ (weak vv-full number $f_{w v v}=f_{w v v}(G)$ ) is the order of the maximum strong (weak) vv-full set of $G$. Note that for any tree $T, \gamma_{s v v}(T)=\gamma_{S}(T)$ and $\gamma_{w v v}(T)=\gamma_{w}(T)$.

\section{Example 4.1:}

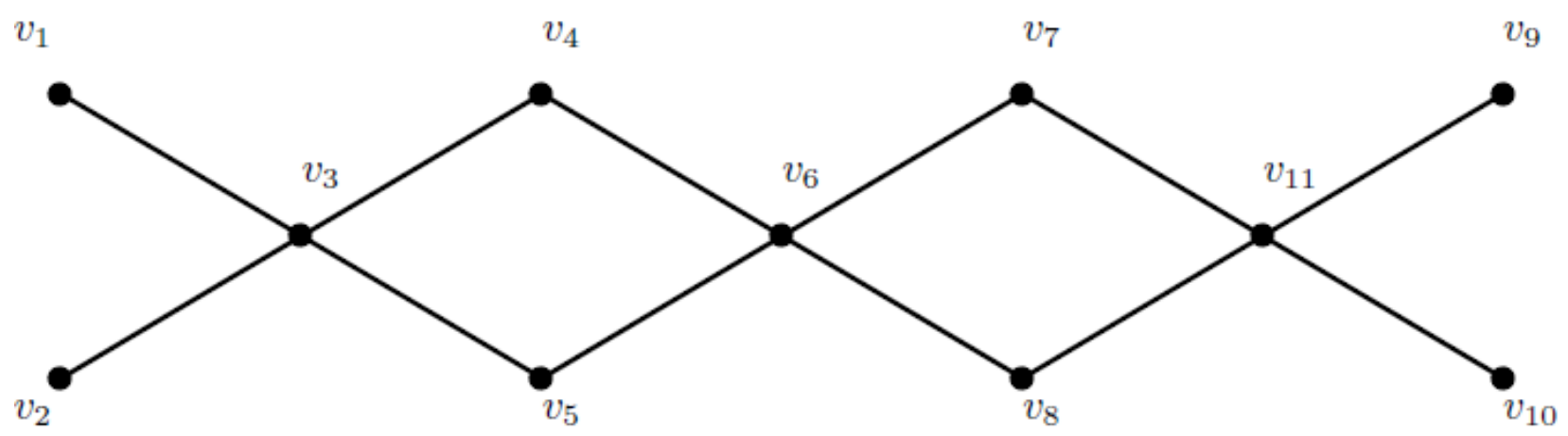

Figure 2. Example for strong and weak vv-dominating sets 
In the Figure 2, VVD-set is $S_{1}=\left\{v_{3}, v_{11}\right\}$, and VVF-set is $V(G)-S_{1}$. Therefore $\gamma_{v v}(G)=2$ and $f_{v v}(G)=9$. Also SVVD-set is $S_{2}=\left\{v_{3}, v_{6}, v_{11}\right\}$ and WVVF-set is $V(G)-S_{2} . \quad$ Therefore $\quad \gamma_{s v v}(G)=$ 3 and $f_{w v v}(G)=8$. Also WVVD-set is $S_{3}=$ $\left\{v_{1}, v_{2}, v_{4}, v_{7}, v_{9}, v_{10}\right\}$ and SVVF-set is
$V(G)-S_{3} . \quad$ Therefore $\quad \gamma_{w v v}(G)=$ 6 and $f_{s v v}(G)=5$

\subsection{Gallai Type Results}

Observation 4.1.1: For any set $D \subseteq V$,

i) $\quad D$ is SVVF-set if, and only if, $V-D$ is a WVVD-set.

ii) $\quad D$ is WVVF-set if, and only if, $V-D$ is a SVVD-set.

Following proposition depicts the relationship between above defined parameters.

Proposition 4.1.2: Let $G$ be the graph with $p$ vertices,

i) $\quad \gamma_{s v v}(G)+f_{w v v}(G)=p$

ii) $\quad \gamma_{w v v}(G)+f_{s v v}(G)=p$.

Proof: Let $S$ be the minimum SVVD set of $G$. Then $V-S$ is an WVVF set by Observation 4.1.1. Hence $f_{w v v}(G) \geq|V-S|$. Therefore $\gamma_{s v v}(G)+f_{w v v}(G) \geq p$. Again, if $D$ is a maximum WVVF set of the graph $G$, then $V-D$ is an SVVD set by Observation 4.1.1. Hence $\gamma_{s v v}(G)+f_{w v v}(G) \leq p$. Then from the above inequalities (i) follows. With similar arguments we can prove (ii).
A block $h$ is called a pendant block if $h$ is incident on a single cutvertex. Nonpendant block which is adjacent to a pendant block is called support block.

A cutvertex $c$ is called an endcutvertex if $c$ is not a cutvertex in the new graph obtained by removing all the pendant blocks. Note that every graph $G$ with $n \geq 1$ cutvertices has at least one end-cutvertex

Proposition 4.1.3 : For any graph $G$

$$
\begin{array}{ll}
\text { i) } & \gamma_{s v v}(G) \leq \gamma_{w v v}(G), \\
\text { ii) } & f_{s v v}(G) \leq f_{w v v}(G) .
\end{array}
$$

Proof: Proof is by principle of mathematical induction on number of cutvertices $n$ of $G$. If $G$ contains only one cutvertex, then clearly $\gamma_{s v v}(G)=1$ and $\gamma_{w v v}(G)=m \geq 2$. where $m$ is the number of blocks in $G$. Therefore $\gamma_{s v v}(G)<\gamma_{w v v}(G)$. Assume that the result is true for $n=k$.. Consider a graph $G$ with $n=$ $k+1$ cutvertices. Let $c$ be an end-cutvertex of $G$ and $b_{1}, b_{2}, \ldots b_{r}$ be the pendant blocks incident on the cutvertex $c$ and $w_{i}$ be a noncutvertex incident on the pendant block $b_{i}$ for $1 \leq i \leq r$. Now, removal of all pendant blocks incident on the end-cutvertex $c$ from $G$ results in a graph $G^{\prime}$ with $k$ cutvertices. Let $W^{\prime}$ be $\gamma_{w v v}$-set of $G^{\prime}$. Then $W=W^{\prime} \cup$ $\left\{w_{1}, w_{2}, \ldots w_{r}\right\}$ is the $\gamma_{w v v}$-set of $G$. Therefore $\gamma_{w v v}(G)=|W|=\left|W^{\prime}\right|+r>\left|W^{\prime}\right|=$ $\gamma_{w v v}\left(G^{\prime}\right)$. Let $S^{\prime}$ be the $\gamma_{s v v}$-set of $G^{\prime}$. Then 
$S=S^{\prime} \cup\{c\}$ is the $\gamma_{s v v^{-s e t}}$ of $G$. Since $G^{\prime}$ has $k$ cutvertices, by induction hypothesis, $\left|S^{\prime}\right| \leq$ $\left|W^{\prime}\right|$. Therefore $\gamma_{s v v}(G)=|S|=\left|S^{\prime}\right|+$ $1 \leq\left|W^{\prime}\right|+1 \leq|W|=\gamma_{w v v}(G)$. Hence the result is true for $n=k+1$. Hence by induction, result is true for all the values of $n$.

The result (ii) follows from the Proposition 4.1.2.

\subsection{Bounds on strong (weak) vv- dominating sets}

We now obtain some elementary bounds for $\gamma_{s v v}(G)$ and $\gamma_{w v v}(G)$. Let $\delta_{v v}(G)$ and $\Delta_{v v}(G)$ respectively be the minimum and maximum vv-degrees of a graph $G$. For any $u \in V, N_{v v}(u)=\{w \in$ $V \mid w$ is vv - adjacent to $u\}$.

Proposition 4.2.1: Let $G$ be any graph with $p$ vertices,

$$
\begin{array}{ll}
\text { i) } & \gamma_{v v}(G) \leq \gamma_{s v v}(G) \leq p-\Delta_{v v}(G) \\
\text { ii) } & \gamma_{v v}(G) \leq \gamma_{w v v}(G) \leq p-\delta_{v v}(G)
\end{array}
$$

Proof: Since every SVVD-set or WVVD-set is a VVD-set, then we have $\gamma_{v v}(G) \leq$ $\gamma_{s v v}(G)$ and $\gamma_{v v}(G) \leq \gamma_{w v v}(G)$. For any $u, w \in V(G)$, let $d_{v v}(u)=\Delta_{v v}(G)$ and $d_{v v}(w)=\delta_{v v}(G)$. It is clear that $V-N_{v v}(u)$ is a SVVD-set and $V-N_{v v}(w)$ is a WVVDset. Hence bound in (i) and (ii) follows.

In the next proposition we obtain a bound for $\gamma_{w v v}(G)$ and characterize the graphs for which the bound is attained.

Proposition 4.2.2: Let $G$ be the graph with $m$ blocks, then $\gamma_{w v v}(G) \leq m$.

Further, $\gamma_{w v v}(G)=m$ if, and only if, there is no cutvertex weakly $v v$-dominates all the vertices incident in at least two blocks.

Proof : In order to weakly vv-dominate all the vertices of $G$, we need at most one vertex $u$ incident on every block $b$ with $d_{v v}(u) \leq$ $d_{v v}(w)$ for every $w$ in $b$ vv-adjacent to $u$. Thus $\quad \gamma_{w v v}(G) \leq m$. Now, $\quad$ suppose $\gamma_{w v v}(G)<m$ and let $W$ be a $\gamma_{w v v}$-set. Then there is some vertex $u$ in $W$ that weakly vvdominates all the vertices of at least two distinct blocks of $G$, a contradiction. Conversely, suppose that $\gamma_{w v v}(G)=m$ Then, there is a minimum WVVD set $W$ of cardinality $m$. Let $W=\left\{w_{1}, w_{2}, \ldots w_{m}\right\}$ are such that $w_{i}$ weakly $b$-dominate the block $b_{i}$ for $1 \leq i \leq m$. Now, suppose $G$ has a cutvertex $u$ which weakly vv-dominates all the vertices incident in two distinct blocks, say $b_{1}$ and $b_{2}$. Then $W^{\prime}=(W-$ $\left.\left\{w_{1}, w_{2}\right\}\right) \cup\{u\}$ is a WVVD set of $G$. Therefore $\quad \gamma_{w v v}(G)=\left|W^{\prime}\right| \leq|W|-2+$ $1=m-1<m$, which is a contradiction.

A cutvertex $k$ is called support cutvertex if $k$ is incident with a pendant block, otherwise it is called non-support cutvertex.

In the next proposition we obtain a bound for $\gamma_{s v v}(G)$ and characterize the graphs for which the bound is attained.

Proposition 4.2.3 : For any graph $G=$ $(V, E)$ with $n \geq 1$ cutvertices, $\gamma_{s v v}(G) \leq n$. Further, $\gamma_{s v v}(G)=n$ if, and only if, there is no non-support cutvertex $u$ such that $d_{v v}(u) \leq d_{v v}(w)$ for at least one cutvertex $w \neq u$ in every block incident with $u$. 
Proof: It is immediate that the set of all cutvertices $S=\left\{v_{1}, v_{2}, \ldots v_{n}\right\}$ is the SVVD set of the graph $G$. Therefore $\gamma_{s v v}(G) \leq$ $|S|=n$. Now, to prove the equality, suppose there is such a non support cutvertex $u$ and let $S$ be the set of all cutvertices of $G$ other than $u$. It is clear that vertices incident with any block not incident with $u$ is strongly vvdominated by a vertex in $S$. Let $b$ be a block incident with $u$. Then by our assumption, there is some cutvertex $v$ different from $u$ having maximum vv-degree in the block $b$, and which therefore strongly vv-dominates all the vertices incident with $b$. Thus $\gamma_{s v v}(G) \leq|S|<n$.

Conversely, if $\gamma_{s v v}(G)<n$, then there is a SVVD set $S$ and at least one cutvertex $u$ not in $S$. Then clearly $u$ is a non-support cutvertex. Further since vertices of every block incident with $u$ is strongly $\mathrm{vv}$ dominated by a vertex $w$ in $S$, we have $w \neq$ $u$ and $d_{v v}(u) \leq d_{v v}(w)$.

For any vertex $u \in V(G)$, vb-degree $d_{v b}(u)$ is the number of blocks incident on $u$.

Since $d_{v b}(u)=1$ for any non cutvertex $u$. We have $\delta_{v b}(G)=1$. we introduce another parameter $\delta_{c v b}(G)=\min _{c \in C(G)} d_{v b}(c)$.

A graph $G$ with $m$ blocks is said to be B-complete if every blocks are adjacent to each other and is denoted by $B_{m}$. In the following corollary we will get an upper bound for $\gamma_{s v v}(G)$ in terms of the number of blocks and $\delta_{c v b}(G)$.

Corollary 4.2.4: Let $G$ be the graph with $m \geq 2$ blocks,

$$
\gamma_{s v v}(G) \leq \frac{m-1}{\delta_{c v b}(G)-1} \text { and equality holds for block complete graph } B_{m}
$$

Proof: It is known that $\sum_{w \in V}\left(d_{v b}(w)-1\right)=$ $m-1$ (Gallai, 1959). Clearly $d_{v b}(w)=1$ for any non cutvertices, $\sum_{k \in C(G)} d_{v b}(k)=$ $m+n-1 \geq n \delta_{c v b}(G)$. Therefore $n \leq$ $\frac{m-1}{\delta_{c v b}(G)-1}$. Also we know that $\gamma_{s v v}(G) \leq n$ from the Proposition 4.2.3. Hence $\gamma_{s v v}(G) \leq$ $\frac{m-1}{\delta_{c v b}(G)-1}$.
For any B-complete graph $B_{m}$ with $m$ blocks, $\delta_{c v b}\left(B_{m}\right)=m$. Therefore $\gamma_{s v v}(G)=1=$ $\frac{m-1}{m-1}=\frac{m-1}{\delta_{c v b}(G)-1}$. Hence equality holds for any B-complete graph $B_{m}$.

Proposition 4.2.5: Let $G$ be the graph with $n^{\prime}$ support cutvertices and e non cutvertices in the pendant blocks, $m_{p}$ pendant blocks and $p$ vertices.

$$
\begin{array}{ll}
\text { i) } & n^{\prime} \leq \gamma_{v v}(G) \leq \gamma_{s v v}(G) \leq p-e \\
\text { ii) } & m_{p} \leq \gamma_{w v v}(G) \leq p-n
\end{array}
$$

Proof: For any graph $G$, there always exists a minimum VVD-set containing all the support cutvertices. Hence $n^{\prime} \leq \gamma_{v v}(G) \leq \gamma_{s v v}(G)$.
Since support cutvertices are sufficient to strongly vv-dominate all the vertices in the pendant block, we have $\gamma_{s v v}(G) \leq p-e$. To 
prove $i i)$, in order to weakly vv-dominates all the vertices of $G$, we need to have at least one vertex from every pendant block. Therefore $\gamma_{w v v}(G) \geq m_{p}$. Also observe that set of all vertices other than support cutvertices forms a WVVD set. Therefore $\gamma_{w v v}(G) \leq p-n^{\prime}$.

A graph $G$ is said to be block path (Bpath) if block graph of $G$ is a path. The length of the B-path is the number of cutvertices in it. For any two blocks $b_{1}, b_{2} \in B(G)$, the Bdistance $d\left(b_{1}, b_{2}\right)$ is the length of the B-path from $b_{1}$ to $b_{2}$. The B-eccentricity of a block $h \in B(G)$ is defined as $e(h)=$ $\max _{g \in B(G)} d(h, g)$. Then B-diameter $d_{b}(G)=$ $\max _{h \in B(G)} e(h)$ and B-radius $r_{b}(G)=$ $\min _{h \in B(G)} e(h)$. A lower bound for $\mathrm{vb}-$ domination number is provided in terms of Bdiameter in our next result.

Proposition 4.2.6: Let $G$ be the graph with $B$-diameter $d_{b}(G)$,

$$
\gamma_{v v}(G)=\frac{d_{b}(G)+1}{2}
$$

Proof : Let $S$ be the $\gamma_{v v}$-set of a connected graph $G$. Consider an arbitrary B-path of length $d_{b}(G)$. Every vertex $v \in S$, can vvdominate at most vertices of two blocks of diametrical B-path. Therefore this B-path includes at most $2 \gamma_{v v}(G)$ blocks and hence $2 \gamma_{v v}(G)-1$ cutvertices. Thus $d_{b}(G) \leq$ $2 \gamma_{v v}(G)-1$ which yields the desired lower bound.

\section{a. Point graph and sum of vv-degree}

A point graph of the graph $G$ denoted as $P_{G}(G)$ is the graph whose vertex set is same as that of the graph $G$ and any two vertices in the point graph are adjacent if and only if they are vv-adjacent in the graph $G$. Observe that $P_{G}\left(P_{G}(G)\right)=P_{G}(G)$. Number of edges in the point graph is denoted as $q_{p}$. For any tree $T, P_{G}(T)=T$. Block-vertex degree (bv-degree) of a block $h, d_{b v}(h)$ is the number of vertices in the block $h$. Cutvertex degree of a block $h, d_{c}(h)$ is the number of cutvertices incident on $h$.

Proposition 4.3.1: Let $G$ be a graph with $m$ blocks and $p$ vertices. Then

$$
\sum_{h \in B(G)} d_{b v}(h)=p+m-1
$$

Proof: It is well known that $\sum_{b \in B(G)}\left(d_{c}(b)-\right.$ 1) $=n-1$ (Harary, 1969). Observe that $d_{b v}(b)=d_{c}(b)+$ number of non cutvertices of the graph $G$. Noting that there are $p-n$ non cutvertices in any graph, we have

$$
\begin{aligned}
& \sum_{h \in B(G)} d_{b v}(h)=\sum_{h \in B(G)} d_{c}(h)+(p- \\
& n)=m+n-1+p-n=p+m-1 .
\end{aligned}
$$

An expression for the number of edges in the point graph in terms of bv-degree is obtained in our next result. 
Proposition 4.3.2: Let $G$ be a graph with $p$ vertices and $q_{p}$ edges. Then

$$
\sum_{w \in V} d_{v v}(w)=2 q_{p}=\sum_{b \in B(G)}\left(d_{b v}(b)\right)^{2}-(p+m-1)
$$

Proof: Since vv-degree of a vertex in $G$ is the degree of the corresponding vertex in $P_{G}(G)$, we $\sum_{w \in V\left(P_{G}(G)\right.} \operatorname{deg}(w)=2 q_{p}$.

As every block in $P_{G}(G)$ is a clique, every block in $G$ yield $\left(\begin{array}{c}d_{b v}(b) \\ 2\end{array}\right)$ edges in the point graph. Then $q_{p}=\sum_{b \in B(G)}\left(\begin{array}{c}d_{b v}(b) \\ 2\end{array}\right)=$ $\frac{1}{2} \sum_{b \in B(G)}\left(\left(d_{b v}(b)\right)^{2}-d_{b v}(b)\right)$. Therefore $2 q_{p}=\sum_{b \in B(G)}\left(d_{b v}(b)\right)^{2}-(p+m-1)$ from the Proposition 4.3.1.

Proposition 4.3.3: For any graph $G$ with $m$ blocks and $p$ vertices,

$$
\sum_{h \in B(G)}\left(d_{b v}(h)\right)^{2} \geq \frac{(p+m-1)^{2}}{m}
$$

Proof: From the Cauchy-Schwarz inequality, we $\sqrt{\sum_{i=1}^{m}\left|a_{i}^{2}\right|} \sqrt{\sum_{i=1}^{m}\left|b_{i}^{2}\right|}$ where $a_{i}$ and $b_{i}$ are integers. Taking $a_{i}=d_{b v}(h)$ and $b_{i}=1$ in the above inequality, we get $\left(\sum_{i=1}^{m} d_{b v}(h)\right)^{2} \leq m \sum_{i=1}^{m}\left(d_{b v}(h)\right)^{2}$. Then the result follows from the Proposition 4.3.1.

\section{CONCLUSION}

Block domination is a well-studied parameter in literature. We modified and studied strong block domination. Few Gallai's theorem type results are obtained. A new class of graphs called point graphs are defined. Several bounds for strong block domination parameters are obtained. Characterization of the graphs attaining these bounds are not studied in full and one may take this as an open problem for further research.

\section{REFERENCES}

Berge, C. (1962) Theory of Graphs and its Applications, Methuen, London.

Bhat, P.G., Bhat, R.S. \& Bhat, Surekha R. (2013). Relationship between Block Domination parameters of a graph, Discrete Mathematics,Algorithms and Applications, 5(3) : 181- 190.

Gallai, T. (1959). Uber extreme Punkt-und Kantenmengen, 133-138, Ann. Univ. Sci.Budapset, Eotvos Sect. Math. 2.

Harary, F. (1969). Graph Theory, Addison Wesley.

Hattingh J. H. \& Renu Laskar. (1998) On weak domination in graphs, Ars Combinatoria-Waterloo

Then Winnipeg, 49 : 205-216. 
Haynes, T. W., Hedetniemi S. T. \& Slater, P. J. (1998). Fundamentals of Domination in Graphs, Marcel Dekker Newyorks.

Henning, M. A. \& Hattingh J. H. (1998). On strong domination in graphs, Journal of Combinatorial Mathematics and Combinatorial Computing, 26 :7382.

Kamath, S. S. \& Bhat, R. S. (2007). On Strong (Weak) Independent sets and Vertex Coverings of a Graph, Discrete Mathematics, 307 :11361145.
Rautenbach, D. (1998). Domination and Degree, Shaker Verlag, ISBN 38265-3899-4.

Sampathkumar E. \& Pushpa Latha, L. (1996). Strong weak domination and domination balance in a graph, Discrete Mathematics 161: 235-242.

West, D. B. (1996). Introduction to Graph Theory, Prentice Hall. 